\title{
Clotted false lumen: reappraisal of indications for medical management of acute aortic dissection
}

\author{
C J SANDERSON, STUART RICH, POLLy A BEERE, C E ANAGNOSTOPOULOS, \\ JAMES M LEVETT, AND J MARK LAWRENCE
}

\begin{abstract}
From the Departments of Surgery and Medicine, The University of Chicago, Division of Biological Sciences and The Pritzker School of Medicine, Chicago, Illinois, and The Long Beach Memorial Hospital, Long Beach, California, USA
\end{abstract}

\begin{abstract}
Evidence of a clotted false lumen in patients with acute aortic dissection has been considered to be a primary indication for medical rather than surgical therapy. A review of recent publications shows that 14 of 15 such patients survived with medical management. We present three patients who had radiographic, surgical, or necropsy evidence of acute aortic $\vec{\varnothing}$ dissection with a clotted false lumen, who suffered further dissection in spite of adequate medical . therapy. Our experience indicates that this condition is not as stable as it has been considered in the past. Consequently, we believe that great caution should be exercised in the application of medical therapy and in the follow-up of patients who demonstrate evidence of a clotted false lumen in an acute aortic dissection. Surgical treatment is indicated at the earliest sign of clinical or radiographic deterioration during medical therapy.
\end{abstract}

Selection of the appropriate therapy for acute aortic dissections has remained a controversial and difficult problem. Although it was once thought that all such dissections should be surgically treated, Wheat et al in 1965, demonstrated that medical therapy could be successful in appropriately selected patients. ${ }^{1}$ Subsequently, several reports confirmed these findings, ${ }^{2-4}$ and the criteria for separating patients into medical and surgical treatment groups have become more clearly defined. ${ }^{4-5}$ The suggested presence of a clotted false lumen on aortography has been generally accepted as one of the primary indications for medical therapy. ${ }^{2-9}$ In this report we describe three patients in whom the presence of a clotted false lumen was suggested angiographically and for whom medical management was eventually unsuccessful. The clinical course of these cases suggests that the indications for medical therapy of acute aortic dissection with clotted false lumen should be re-examined.

Address for reprint requests: Dr CE Anagnostopoulos, The University of Chicago, 950 East 59th Street, BH E508 Box 152, Chicago, Illinois 60637, USA.

\section{Case reports}

CASE 1

A 67-year-old hypertensive white woman was re- $\stackrel{0}{x}$ ferred to the University of Chicago Hospitals with $\frac{0}{3}$ a 24-hour history of severe back pain. On physical examination the blood pressure was $130 / 100$, the right carotid pulse was diminished, and there were bruits over both carotid arteries, both external 을 iliac arteries, and the abdominal aorta. A chest $\rightarrow$ radiograph demonstrated widening of the mediastinum and a left-sided pleural effusion. Emer- $N$ gency aortography suggested a diagnosis of acute aortic dissection (De Bakey type I) with a clotted false lumen (fig 1).

A thoracocentesis yielded bloody fluid and ${ }^{\circ}$ treatment was started with antihypertensive agents $\stackrel{\bullet}{\subset}$ and diuretics. On the second day of treatment the $\stackrel{\mathscr{D}}{\mathscr{D}}$ patient had a pulmonary embolus (confirmed by $\stackrel{+}{-}$ angiography) and an inferior vena caval filter was inserted.

The blood pressure, which was initially very $\frac{\mathbb{Q}}{\mathbb{D}}$ labile, slowly came under control and her con- $\unrhd$ dition stabilised. Serial chest radiographs failed to show any alteration in the mediastinum. 
On the thirty-third day of treatment the patient experienced another episode of severe back and chest pain followed by dyspnoea and the blood pressure once again became very unstable. A chest radiograph showed no appreciable change in the appearance of the aorta compared with previous films (fig 2). Shortly afterwards the patient became hypotensive and had a cardiac arrest from which she could not be resuscitated.

Postmortem examination revealed a dissection of the thoracoabdominal aorta. The false lumen contained multiple clots in varying stages of organisation (fig 3 ).

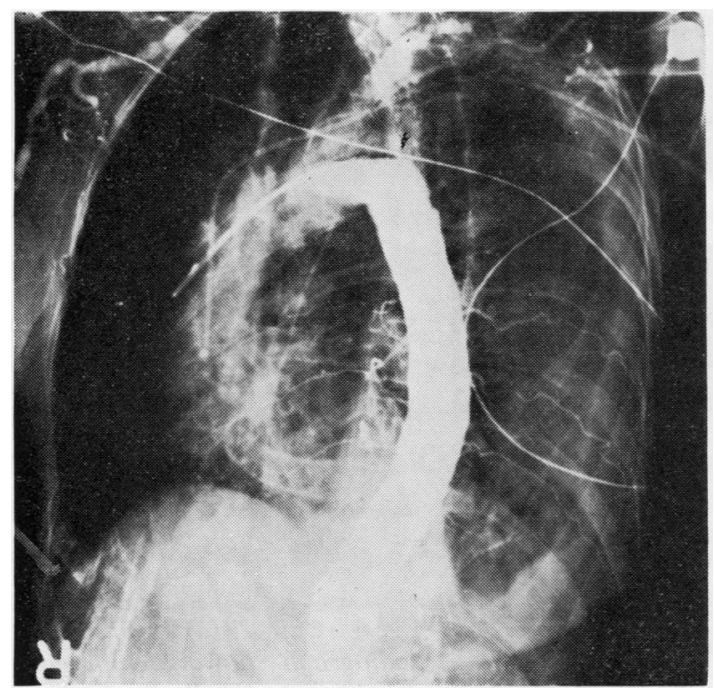

Fig 1 Case 1. Aortogram demonstrating density around compressed aortic lumen and an angular deformity (arrow) visualised distal to the subclavian artery.
CASE 2

A severely hypertensive 47-year-old black man was admitted to The University of Chicagn Hospitals with back pain. Physical examination was unremarkable. A chest radiograph demonstrated a widened aortic shadow (fig 4). Emergency aortography confirmed the clinical diagnosis of acute aortic dissection (De Bakey type III) with a clotted false lumen (fig 5). The patient responded well to treatment with antihypertensives and diuretics and was discharged after 20 days in hospital.

Nine months later he was readmitted with recurrent symptoms. On examination the left leg pulses were markedly diminished. The chest radiograph (fig 4) and aortogram (fig 6) demonstrated an increase in size of the aortic knuckle and expansion of the previously clotted false lumen.

The patient underwent successful surgical repair of the aortic dissection. Intraoperative exploration verified the presence of a medial dissection of the thoracic aorta with thrombus in the false lumen. The postoperative course was uncomplicated and the patient was discharged in good condition. He continues well 18 months after the procedure.

CASE 3

A 74-year-old white man was admitted to Long Beach Memorial Hospital with a two-hour history of severe back pain. There had been no significant previous illnesses. The patient's blood pressure was $165 / 100$ but otherwise physical examination was unremarkable. A chest radiograph demonstrated an enlarged aortic knuckle (fig 7). An emergency aortogram revealed dissection of the thoracic aorta (De Bakey type III) with a clotted false lumen (fig 8). Antihypertensive

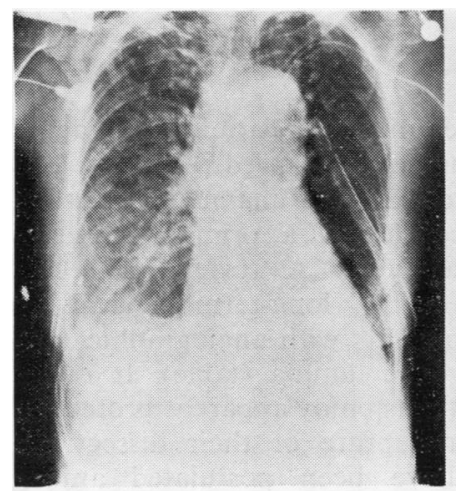

(a)

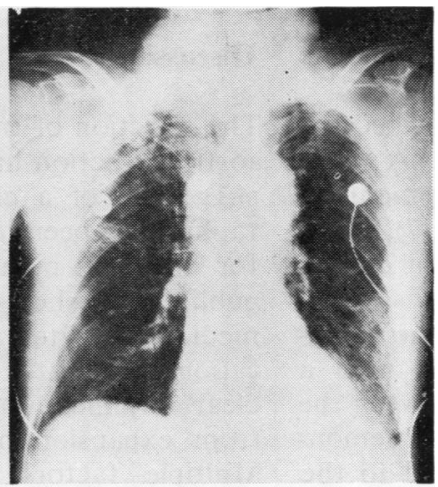

(b)
Fig 2 Case 1 (a) Admission radiograph. (b) Radiograph immediately before death. No significant change in the mediastinal shadows is evident. 

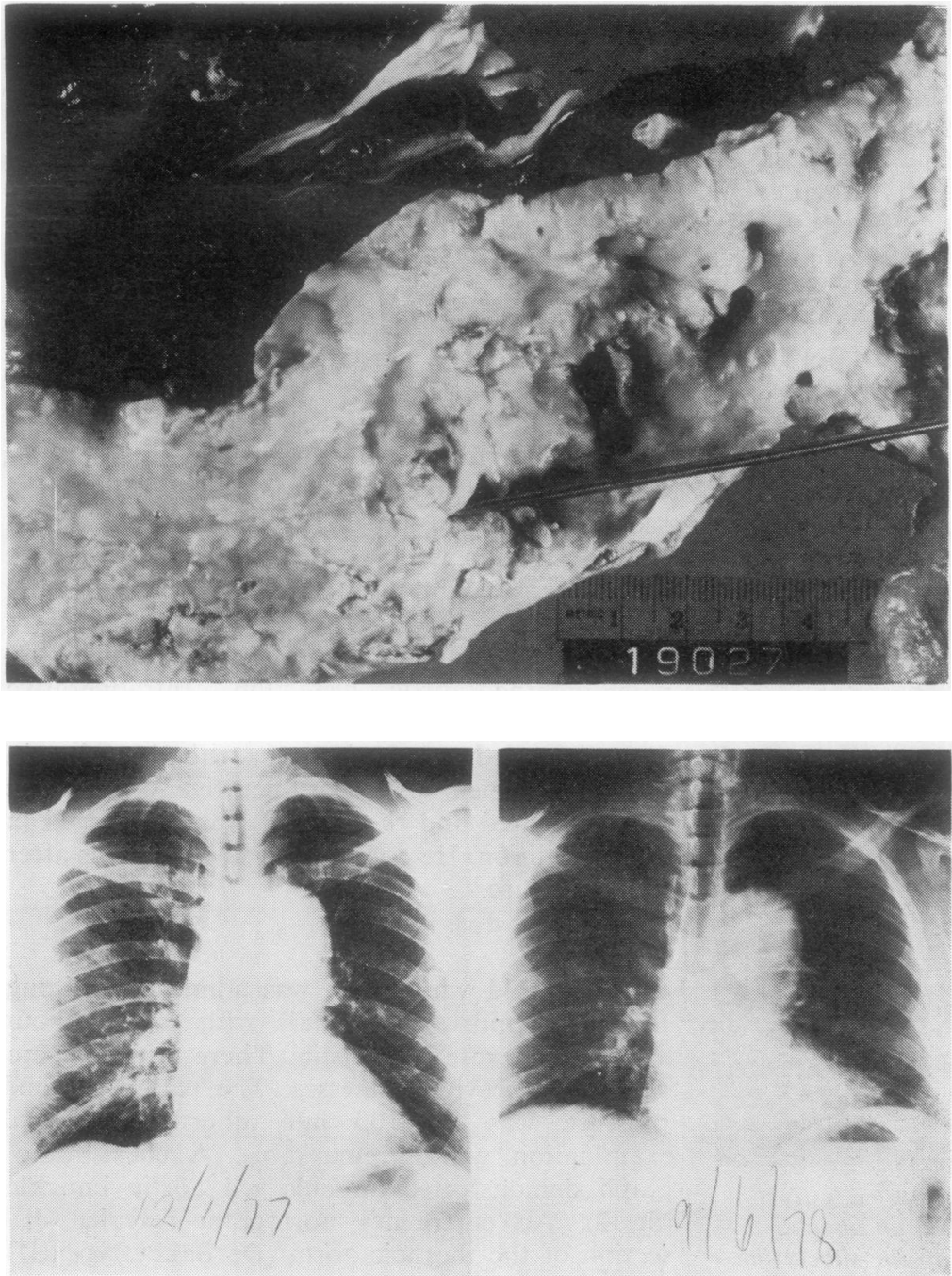

(a)

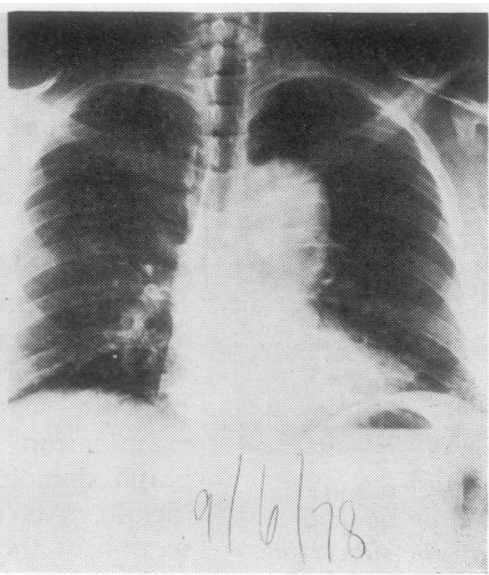

(b)
Fig 3 Case 1. Gross specimen of thoracic aorta showing luminal tear probe and multiple clots (arrows)
Fig 4 Case 2. (a) Initial radiograph showing enlarged aortic arch. (b) Radiograph after nine months of medical treatment showing a further increase in size of aortic arch. treatment relieved the patient's symptoms and he was discharged after 30 days in hospital.

Three weeks after discharge the patient became hoarse and was readmitted to hospital. A chest radiograph showed considerable enlargement of the aortic arch and descending aorta (fig 7). However, an aortogram again demonstrated a clotted false lumen (fig 8).

Exploration confirmed the findings of the aortogram. The thoracic aorta was replaced with a tubular graft but the patient died suddenly on the eighth day after the operation. A necropsy demonstrated a new dissection originating distal to the graft. The surgical repair was satisfactory.

\section{Discussion}

The selection of treatment for patients with acute $\tilde{乛}$ aortic dissection has been reviewed recently. ${ }^{6}$ The suggestion of a clotted false channel on aortography has been considered a primary criterion $c$ for medical management. A review of recent $\mathbb{D}$ publications shows a 93\% long-term survival for medically treated patients with angiographic indications of clotted false lumina (table). It is not clear why these patients enjoy apparent protection from expansion or rupture of their dissections. Multiple factors have been postulated among which is the suggestion that the thrombosed 
channel provides a buttress against further rupture or extension.

In contrast to the above observations, our three patients with confirmed clotted false lumina failed to respond to medical therapy alone, thus challenging the previously held view on management of such patients. There are several reasons why this discrepancy. might exist. First, the angiographic criteria for diagnosis of a clotted false lumen are ill-defined. ${ }^{712} \mathrm{It}$ is possible that patients with a clotted false lumen who have had a second dissection may not have been diagnosed accurately

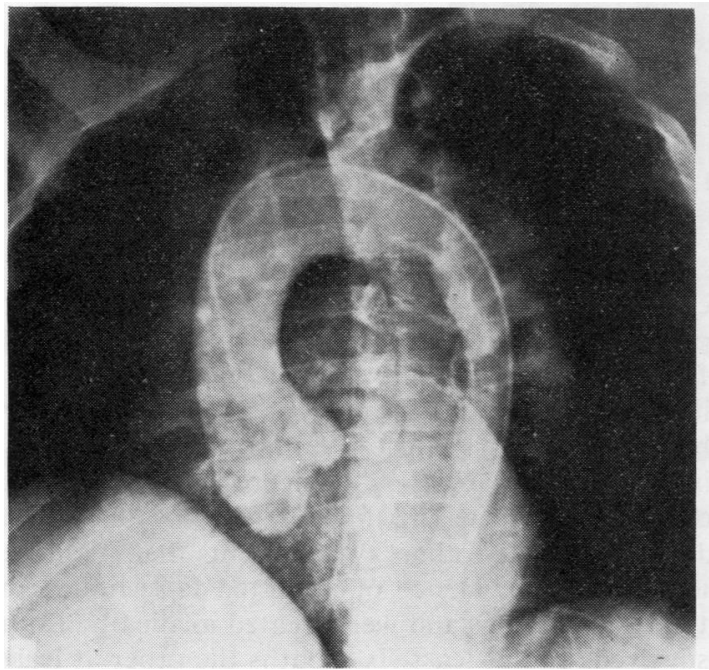

Fig 5 Case 2. Aortogram during first admission demonstrating abnormal compression of the descending thoracic aorta originating at the distal aortic arch.
Table Review of publications on medical treatment for patients with clotted false lumen

\begin{tabular}{lcc}
\hline & $\begin{array}{c}\text { Patients with clotted } \\
\text { false lumina }\end{array}$ & Survived \\
\hline Dinsmore $^{7}$ & 10 & 9 \\
Wheat $^{8}$ & 1 & 1 \\
Thomas $^{9}$ & 1 & 1 \\
Anagnostopoulos $^{5}$ & 3 & 3 \\
Total & 15 & 14 \\
\hline
\end{tabular}

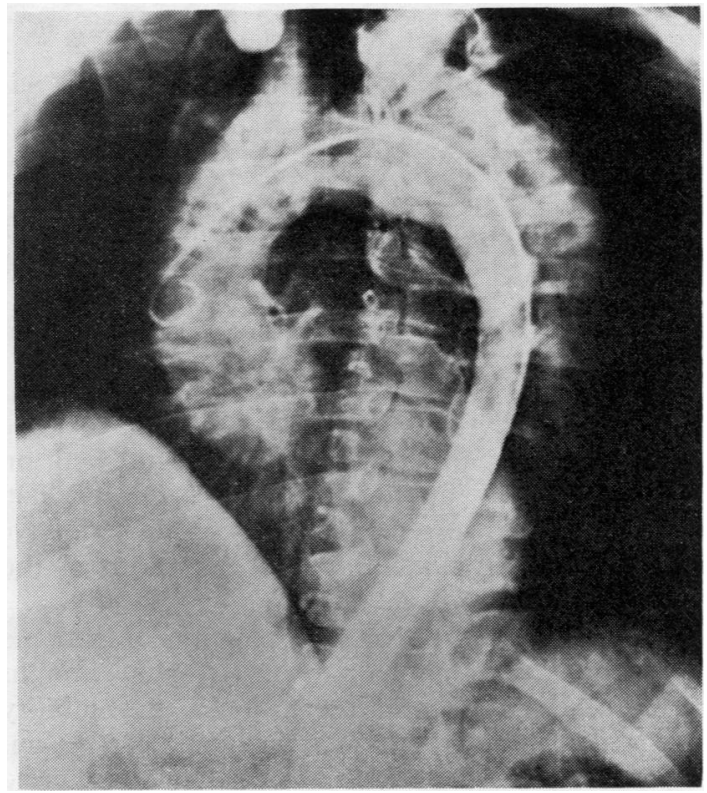

Fig 6 Case 2. Aortogram at the time of the second admission revealing entry of contrast material into the false lumen which extends from the subclavian artery to the mid-thorax.

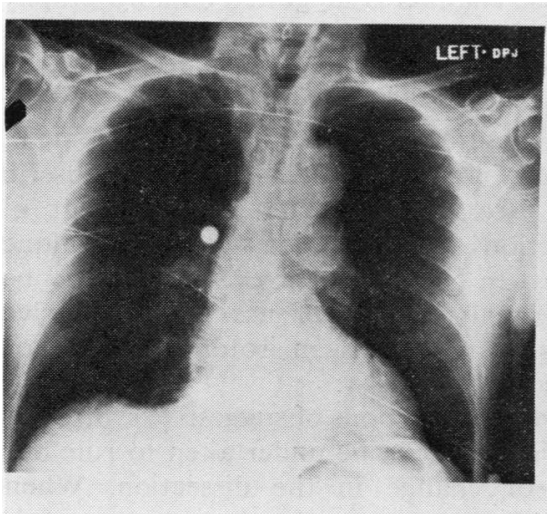

(a)

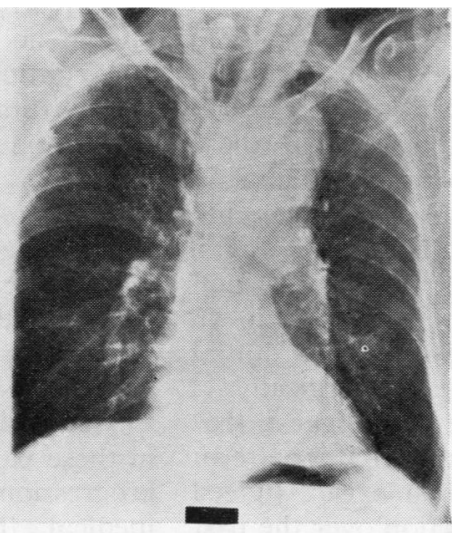

(b)
Fig 7 Case 3. (a) Chest radiograph on first admission showing enlarged aortic arch. (b) Chest radiograph at the time of the second admission six weeks later showing increased enlargement of aortic arch and mediastinal silhouette. 


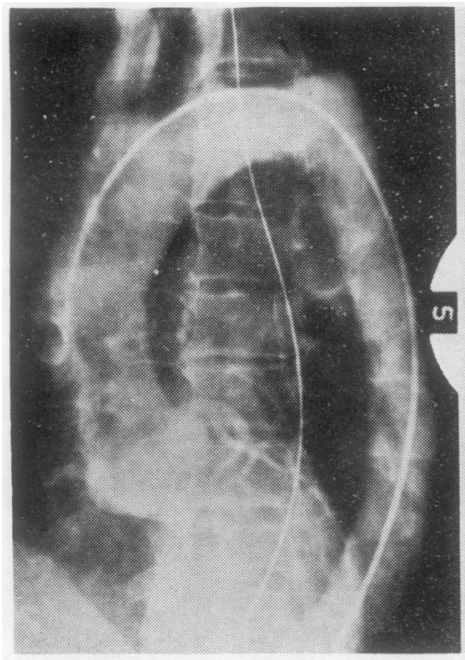

(a)

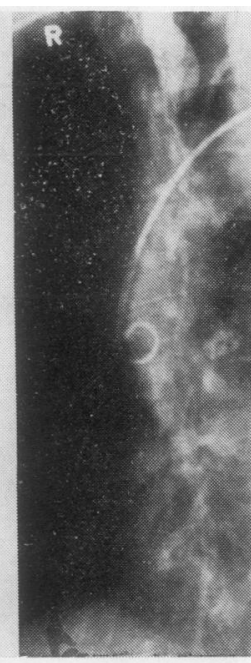

(b)
Fig 8 Case 3. (a) Aortogram on furst admission demonstrating angular deformity of distal aortic arch (arrow) and compression of thoracic aortic lumen.

(b) Aortogram at second admission showing an increase in size of angular deformity and compression of descending aortic lumen. originally. Conversely, patients believed to have clotted false channels who have done well on medical therapy might also have been misdiagnosed. Our present series is unique in that we have surgical or necropsy confirmation or both of the dissection process in all cases.

It is also possible that the selection of patients for medical treatment as previously reported might be biased, giving a false impression of the stability of the clotted false channel in aortic dissection. To our knowledge, there has been no randomised trial of medical versus surgical treatment in these patients.

The cases presented here demonstrate that expansion of the original dissection and new dissection are possible in patients with clotted false lumina despite apparently successful medical therapy. In view of this, we feel that decisions to treat these patients medically should undergo careful scrutiny. This is not to say that medical treatment has no role in the management of these patients. The inclusion of our three patients in the above quoted series still gives a survival rate of $78 \%$ for the medically treated group. In all three cases, the subsequent crisis was an extension of the previous dissection, but in addition, in case 3, a new dissection arose distal to the initial site of disease which had been treated surgically.

We have no evidence as yet to suggest that surgical treatment will lead to a better long-term result when a clotted false lumen exists. Indeed in a review of acute aortic dissection over the past 10 years in this institution which we are at present completing, we have uncovered three further patients who had definite evidence of a clotted false lumen. Of these three, one was severely hypotensive on arrival and was treated medically but died 24 hours later of rupture of the descending thoracic aorta. At necropsy there was organised clot in the false lumen of a type 1 dissection. The other two patients had definite angiographic evidence of a clotted false lumen in type 3 dissections and were treated medically. Both survived, one at least six months the other at least six years. The outcome of case 1 and the first case mentioned here could not have been worse had surgery been performed.

We now advise, however, that patients with acute aortic dissection in whom the presence of a clotted false channel is suggested on aortography should no longer be considered prime candidates for continuous medical treatment. After initial stabilisation frequent assessment should be made to provide assurance of successful treatment. Although aortography remains the most useful method of assessing the nature and extent of aortic dissections, the limitations of this technique are apparent. In future, better evaluation may be available through the use of newer non-invasive techniques such as fibrinogen isotope scanning or computerised tomography.

Aggressive investigations of suggestive symptoms in these patients should be undertaken to rule out progression or change in the dissection. When medical therapy appears to be unsuccessful, surgical treatment should be instituted promptly. 
This work was supported by The Combined Cardiac Surgery Research Fund of the University of Chicago.

\section{References}

1 Wheat MW, Palmer RF, Bartley TD et al. Treatment of dissecting aneurysms of the aorta without surgery. J Thorac Cardiovasc Surg 1965; 50: 364-73.

2 McFarland J, Willerson JT, Dinsmore RE et al. The medical treatment of dissecting aortic aneurysms. N Engl J Med 1972; 286:115-9.

3 Dalen JE, Alpert JS, Cohn LH, Black H, Collins JJ. Dissection of the thoracic aorta. Am J Cardiol 1974; 34:803-8.

4 Anagnostopoulos CE. Acute aortic dissections. Baltimore: University Park Press, 1975.

5 Kolff J, Bates RJ, Balderman SC, Shenkoya K, Anagnostopoulos CE. Acute aortic arch dissection: reevaluation of the indications for medical and surgical therapy. Am J Cardiol 1977; 39:
727-33.

6 Anagnostopoulos CE. Lethal diseases of the descending aorta. Baltimore: University Park Press, 1976.

7 Dinsmore RE, Willerson JT, Buckley MJ. Dissecting aneurysm of the aorta. Radiology 1972; 105: 567-72.

8 Wheat MW. Treatment of dissecting aneurysms of the aorta: current status. Prog Cardiovasc Dis $1973 ; 16: 87-101$.

9 Thomas CS, Alford WC, Burrus GR, Frist RA, Stoney WS. The effectiveness of surgical treatment of acute aortic dissection. Ann Thorac Surg 1978; 28:42-9.

10 Miller DC, Stinson EB, Oyer PE et al. Realistic expectations following aggressive surgical treatment of acute and chronic aortic dissectionsexperience with 111 patients over a 14 year period. Am Assoc Thorac Surg Proc, 1979.

11 Earnest F, Muhm JR, Sheedy PF. Roentgenographic findings in thoracic aortic dissection. Mayo Clinic Proc 1979; 54:43-50. 